\title{
Fuzzy Logic Adaptive Online Optimization of Sliding Mode Control with Boundary Layer Self-tuning
}

\author{
Jingxin Wei and Xiaoyu Zhang* \\ School of Electronic and Information Engineering, North China Institute of \\ Science and Technology, Beijing 065201, China \\ weijing7703@ncist.edu.cn; ysuzxy@aliyun.com
}

\begin{abstract}
In this paper, we present a fuzzy logic adaptive online optimization method with a sliding mode control (SMC) and boundary layer self-tuning based on an approximate mode for nonlinear discrete systems. First, parameters of the sliding mode boundary layer are made with online adjustments through some adaptive law to get smooth sliding mode control. Second, a fuzzy logic system whose structure and rules take online optimization is applied to equivalent SMC with the boundary layer self-tuning, which includes the improvement of a cluster-learning algorithm. Simulation results have proved the correctness and superiority of the proposed online optimization method for the fuzzy logic system.
\end{abstract}

Keywords: fuzzy logic system, optimization, sliding mode control, boundary layer, discrete nonlinear systems

\section{Introduction}

Fuzzy logic system (FLS) is widely applied [1], especially to the plants that precise mathematics models cannot easily acquire. Based on the application of FLS, many adaptive sliding mode control (ASMC) approaches are deeply investigated recently. A T$S$ type fuzzy system is applied to describe the origin nonlinear system in [2], and for nonlinear active suspension systems with sprung and unsprung masses, the unknown actuator nonlinearity and the suspension performances are taken into account simultaneously. Then an ASMC controller is designed to guarantee the reachability of the specified switching surface [2]. The same frame work is presented in [3]. Both state and static output feedback SMC control designs are considered and results are given in terms of linear-matrix inequalities (LMIs). The development of an adaptive fuzzy sliding mode controller (AFSMC) for an electro-hydraulic system with unknown dead-zone is proposed by [4], in which the boundedness and convergence properties of the closed-loop signals are proven using Lyapunov stability theory and Barbalat's lemma. An enhanced selforganizing fuzzy sliding-mode controller for robotic systems is developed by [5], which uses a sliding surface and its differentiation as the input variables of a FLS. It also employs an adaptive law to modify the fuzzy consequent parameter. An enhanced indirect adaptive fuzzy sliding mode based power system stabilizer for damping local and interarea modes of oscillations is presented for multi-machine power systems [6], in which generator speed deviation and its derivative are selected as input signals to a FLS that approximates unknown power system functions and adaptation laws are developed. An AFSMC is also proposed to deal with a class of uncertain chaotic systems subject to an

\footnotetext{
* Corresponding Author. This work was supported by National Natural Science Foundation of China (61304024), the Science and Technology Project of Hebei Province (15272118), the Fundamental Research Funds for the Central Universities (3142016022) and the Science and Technology Research and Development Program of Langfang City (2016011006).
} 
unknown dead-zone input [7]. Nekoukar presented an AFSMC via a terminal sliding mode control (TSM) design, which was to provide faster convergence and higherprecision [8]. Yoshimura presented the design of an AFSMC for uncertain discrete-time nonlinear dynamic systems with noisy measurements [9].

In the method of AFSMC, the information of FLS is always redundant. Therefore, many researches concern the optimization of FLS. Chen et al. discussed the stability analysis of a genetic algorithm-based $H_{\infty}$ adaptive fuzzy sliding model controller (AFSMC) for a nonlinear system, in which a FLS is adopted to approximate the nonlinear plant [10]. The FLC rules and the consequent parameter are decided on via a genetic algorithm. In the same frame, Bendaha et al. proposed an optimization technique of fuzzy sliding mode control (FSMC) using genetic algorithm to overcome the shortcoming that fuzzy control rules are always built by designers with trial and error [11]. An optimal FSMC controller is designed using an intelligent fuzzy controller based on Sugeno-Type structure, and is optimized by a novel heuristic algorithm, namely particle swarm optimization with random inertia weight (RNW-PSO) [12]. The same optimization approach is applied to the FSMC control for tracking the position of robot manipulator [13], and the optimization of the parameters of a FSMC, as well as the input and output membership functions [14].

In this paper, the optimization of the fuzzy rules is mainly considered in a typical FLS application to sliding mode control that forms the sliding mode-like fuzzy logic control (SMLFC). Firstly, we have proposed SMLFC for nonlinear systems in paper [18]. As the same idea in papers [13-18], the FLC controller is achieved, which is equivalent to the pre-designed SMC controller with boundary self-tuning. A dynamic fuzzy logic system (DFLS) will then be optimized by cluster-learning algorithms to get better control performance. A practical algorithm will be provided in detail.

We organized this paper as follows: In Section 2, we presented problem formulation and a general SMC. The preliminaries of a SMLFC were approached in Section 3. Then we reported the optimization process and algorithm in Section 4, which will simplify the rules and the inference work of the FLS. Section 5 included proposals of simulation results of a numerical example to validate the controller design. Section 6 concerned a summary of conclusions.

\section{Problem Description and SMC}

In this part, we raise basic SMC control questions concerning one kind of discrete nonlinear system and then design SMC controller. This part of continent is cited from [18].

In view of the following nonlinear system:

$$
\begin{aligned}
& x_{i}(k+1)=x_{i+1}(k), i=1,2, \cdots, n-1 \\
& x_{n}(k+1)=f(x, k)+g(x, k) u(k)
\end{aligned}
$$

where $x=\left[x_{1}(k) \cdots x_{n}(k)\right]^{T}$ is the state vector, $f(x, k)$ is the nonlinear dynamic function, $u(k)$ is the single control input, and $g(x, k)$ is the nonlinear gain of the control input.

It is assumed that the desired trajectory is $y_{d}$ and $x_{d}=\left[x_{d 1}(k) \cdots x_{d n}(k)\right]^{T}$ is the corresponding auxiliary state vector. Suppose that $y_{d}=x_{d 1}(k)$ and $x_{d i}(k)(i=1,2, \cdots, n)$ is bounded for all time interval $k(k \in[0,+\infty))$, which satisfies the stable tracked model:

$$
\begin{aligned}
& x_{d i}(k+1)=x_{d(i+1)}(k),(i=1,2, \cdots, n-1) \\
& x_{d n}(k+1)=-\sum_{i=0}^{n-1} a_{i} x_{d(i+1)}(k)+r(k)
\end{aligned}
$$

The problem is to construct a sliding mode control law $u\left(r(k), x_{d}(k), x(k), s(k), k\right)$ such that the error vector 


$$
e=\left[e_{1}(k) \cdots e_{n}(k)\right]^{T}, e_{i}(k)=e_{i}(k)-e_{d i}(k), i=1,2, \cdots, n
$$

converges to the tolerable range, where $s(k)$ is the sliding mode surface:

$$
s(k)=c^{T} e, c=\left[\begin{array}{llll}
c_{1} & \cdots & c_{n-1} & 1
\end{array}\right]^{T}
$$

and the vector $c$ can make a Hurwitz polynomial.

Suppose we estimate the nonlinear dynamic function $f$ as $\hat{f}(x, k)$, and the nonlinear control gain $g$ as $\hat{g}$, where $\hat{g}$ is a constant scalar. Then the uncertainty $\Delta f$ and $\Delta g$ are defined as:

$$
\Delta f=f(x, k)-\hat{f}(x, k), \quad \Delta g=g(x, k)-\hat{g}
$$

Without loss of generality, suppose that $g(x, k)>0, \hat{g}>0$ and $\Delta \bar{g}$ is the upper boundary of $\Delta g$. Then $\hat{g}^{-1} \Delta g>-1$ holds.

For convenience, define that

$$
\delta=|\Delta f|+\left|\Delta g \cdot u_{e q}\right|, \quad \gamma=1+\hat{g}^{-1} \Delta g
$$

Then $\delta>0,0<\gamma \leq \bar{\gamma}$, with $\bar{\gamma}=1+\hat{g}^{-1} \Delta g$. Here, $u_{e q}$ is the equivalent control of the sliding mode control $u(k)$.

If the SMC control input $u$ is constructed as:

$$
\begin{aligned}
& u(k)=u_{e q}+u_{v} \\
& u_{e q}=\hat{g}^{-1}\left[c x_{d}(k+1)-\sum_{i=1}^{n-1} c_{i} x_{i+1}(k)-\hat{f}(x, k)+s(k)\right] \\
& u_{v}=-\hat{g}^{-1} k_{1} \operatorname{sat}\left(\frac{s(k)}{\phi}\right)
\end{aligned}
$$

where:

$$
\operatorname{sat}\left(\frac{s(k)}{\phi}\right)=\left\{\begin{array}{cc}
s / \phi, \text { if } & 0<|s|<\phi \\
\operatorname{sgn}(s), \text { if } & |s| \geq \phi \\
0, \text { if } \quad s=0
\end{array}\right.
$$

and:

$$
\phi=\delta+\eta+\varepsilon
$$

is the thickness of the boundary layer [18] with $\eta>0$ and $\varepsilon<0$ are positive scalars that can be optional small; $k_{1}$ is given by:

$$
k_{1}=\gamma^{-1}(\delta+\eta)
$$

Then the reaching condition:

$$
\Delta\left[s^{2}(k)\right] \leq-\eta|\Delta s(k)| \text { if } \quad|s(k)|>\phi
$$

can be satisfied by the controller (7) and the system tracking error vector $e$ is bounded.

Theorem 2.1 [18]. As for the error system described in (1) - (3) and its sliding mode (4), under the influence of the sliding mode control (7), (8), the reaching conditions (11) can be satisfied, the switching band of sliding mode $B=\{e(k) \| s(k) \mid \leq \phi\}$ is reachable and stable. That is to say, $\exists k_{s}>0$ makes $\forall k>k_{s}$ get $e(k) \in B$. Further, if $\Delta f=\Delta g=0$, then $s(k)$ asymptotically converges to the ideal sliding surface $\mathbf{S}=\{e(k) \mid s(k)=0\}$.

However, parameters $\gamma$ and $\delta$ are all unknown, so the control (7) cannot be implemented because $k_{1}$ and $\phi$ cannot be determined. In the following section, we will introduce the preliminaries of the method that deals with it. 


\section{Preliminaries of SMFLC}

Supposing that $k_{1}$ can be estimated and the boundary layer $\phi$ that satisfies the equation (9) can be tuned by $\Delta \phi(k)$. Define $\hat{k}_{1}$ is the estimating value of the variable $k_{1}-\Delta \varphi(k)$ and $\tilde{k}_{1}=\hat{k}_{1}-\left[k_{1}-\Delta \varphi(k)\right]$ is the error of the estimation, then the condition (11) should be satisfied. Choose the following adaptive law

$$
\begin{aligned}
& \Delta \hat{k}_{1}(k)=\left\{\begin{array}{l}
\alpha(1+\beta)^{-1}\left[\beta \hat{k}_{1}(k)-\alpha \varphi(k)+s(k)\right], \quad \text { if } \quad|s|>\varphi \\
-\tau|s|, \quad \text { if }|s| \leq \varphi
\end{array}\right. \\
& \Delta \varphi(k)= \begin{cases}-\alpha \varphi(k)+\beta \hat{k}_{1}(k)+s(k), & \text { if }|s|>\varphi \\
-2 \hat{k}_{1}(k), & \text { if } \quad|s| \leq \varphi\end{cases}
\end{aligned}
$$

where $\alpha>0, \beta>0, \tau>1$ are all constant scalars, i.e. the filtering parameters of the adaptive law. Here we assume that $\hat{k}(0)>0, \varphi(0)>0$, and then based on the SMC (7) and the adaptive online tuning scheme (12)(13), the following theorem can be approached.

Theorem 3.1 [18]. For a nonlinear system (1) with the control law (7), the sliding mode (4) reaches to $B^{\prime}=\{e(k) \| s(k) \mid \leq \phi(k)\}$ and the closed-loop system is asymptotically stable if in (7) the control gain $k_{1}$ that is replaced with $\hat{k}_{1}$, and the boundary layer $\phi$ are all of being adaptively tuned online by the adaptive law (12-13).

Consider the same idea in [18], replace the control input $u_{v}$ with the output of the DFLS $\bar{u}_{v}$ :

$$
\bar{u}_{v}=-\omega_{1} \bar{u}_{v}+\omega_{2} \xi^{T} p
$$

where $\omega_{1}>0, \omega_{2}>0$ are filtering parameters of DFLS to be designed. $\xi=\left[\begin{array}{lll}\xi_{1} & \cdots & \xi_{m}\end{array}\right]^{T}$ is the support points vector of the fuzzy rule base and $p=\left[\begin{array}{lll}p_{1} & \cdots & p_{m}\end{array}\right]^{T}$ is the fuzzy rule base function vector which is determined by:

$$
p_{i}=\frac{\prod_{i=1}^{l} u_{i}^{j}}{\sum_{i=1}^{m} \coprod_{j=1}^{l} u_{i}^{j}}
$$

with $l$ as the total number of input variables, $m$ as the total number of fuzzy reasoning rules, and $u_{i}^{j}$ as the value of the membership function of the $j$-th variable in the $i$-th rule. Suppose the fuzzy partition number of the $j$-th input variable is $\xi$ then the number of fuzzy rules $m=\coprod_{j=1}^{l} \xi_{j}$.

The detail of the DFLS design refers to [18].

To approximate the variable structure control input $u_{v}$, the output of the DFLS $\bar{u}_{v}$ must satisfy the relation between $u_{v}$ and $\hat{k}_{1}$ (the equation (7)). So one can calculate $\hat{k}_{1}$ by

$$
\hat{k}_{1}=\frac{\bar{u}_{v}}{\hat{g}^{-1} \operatorname{sat}(s, \phi)}
$$

Then, $\phi$ can be self-tuning by the adaptive law (13) while the adaptive law (12) is not used; $\phi$ is also the partition parameter of the input variables, so the fuzzy inferring with self-tuning is implemented.

The SMLFC control law comprises the sum of $u_{e q}$ and $\bar{u}_{v}$. 


\section{Optimization of the Rules in FLS}

In this part the application of optimization algorithm is the main originality of this paper, which is for the online optimization of the FLS.

The adaptive fuzzy logic system is the combination of a fuzzy system and an online adjustment of the network. The adaptive law is used for online adjustment of the fuzzy logic system parameters to realize the fuzzy inference, so that the generated fuzzy systems get approximate nonlinear control and the network ability of self-learning and adaptive ability. The fuzzy system usually adopts cluster-learning algorithms in the optimization and adjustment of the rules (such as the fuzzy c-means clustering algorithm, k-means method, nearest neighbor clustering algorithm, etc.) to obtain the initial fuzzy control rules, and then gets the parameters in the control rules to the network for parameter adjustments and rule optimization.

Clustering means dividing the collection of data (sample set) into disjointed groups. Data in one group should have some characteristics that distinguish themselves from those in other groups, and dissimilar data samples are classified into different groups. Here, the nearest neighbor clustering algorithm is presented. The nearest neighbor clustering algorithm is one kind of simple clustering algorithm. The first data are taken as the cluster center of the first group, and if data from the cluster center distance is less than a certain expected number, the data are put into this group, or these data will be set as one clustering center for a new set.

\subsection{Control Rules Optimization Online}

To obtain control rules online with the nearest neighbor algorithm, we change the fuzzy rules of the FLS online. The following introduces the steps for designing the fuzzy systems with the control rules obtained by the nearest neighbor clustering algorithm.

Step 1: To start from the first input-output data pair, suppose $x_{0}^{1}$ as a cluster center, and set $A^{1}(1)=y_{0}^{1}, B^{1}(1)=1$ and set $r$, the radius of clustering.

Step 2: To assume that $k$ pair input-output data $\left(x_{0}^{k}, y_{0}^{k}\right), k=2,3, \cdots, M$ clustering exist that have centers as $x_{c}^{1}, x_{c}^{2}, \ldots \ldots x_{c}^{M}$ respectively. To calculate the distance from $x_{0}^{k}$ to these $M$ cluster centers $\left|x_{0}^{k}-x_{c}^{l}\right|, l=1,2, \cdots M$. Among these distances, the minimum is $\left|x_{0}^{k}-x_{c}^{l_{k}}\right|$, which means that $x_{c}^{l_{k}}$ is the clustering of the nearest neighbor principle of $x_{0}^{k}$.

If: (a) $\left|x_{0}^{k}-x_{c}^{l_{k}}\right|>r$,

there exists $x_{c}^{M+1}=x_{0}^{k}$, suppose $x_{0}^{k}$ as a new cluster center, and set $A^{M+1}(k)=y_{0}^{k}$, $B^{M+1}(k)=1, \quad A^{l}(k)=A^{l}(k-1), B^{l}(k)=B^{l}(k-1), l=1,2, \cdots, M$. The known sample data divides into an $M+1$ cluster and each cluster corresponds to a set of input-output data pairs and at the same time produces fuzzy control rules through the above clustering algorithm, so you can construct an initial fuzzy rule base.

Rule $R^{j}$ : if $x_{1}$ is $A_{1}^{j}\left(x_{1}\right), \ldots \ldots$, and $x_{n}$ is $A_{n}^{j}\left(x_{n}\right)$ then $y \ldots \ldots, j=1,2, \ldots, M+1$,

where $M+1$ is number of fuzzy rules, then the FLS designed by this control rule of $M+1$ is:

$$
f_{k}(x)=\frac{\sum_{l=1}^{M+1} A^{l}(k) \exp \left(-\frac{\left|x-x_{c}^{l}\right|^{2}}{\sigma}\right)}{\sum_{l=1}^{M+1} B^{l}(k) \exp \left(-\frac{\left|x-x_{c}^{l}\right|^{2}}{\sigma}\right)}
$$

(b) $\left|x_{0}^{k}-x_{c}^{l_{k}}\right|<r$, 
$x_{0}^{k}$ is bound to clustering $l_{k}, A^{l_{k}}(k)=A^{l_{k}}(k-1)+y_{0}^{k}, B^{l_{k}}(k)=B^{l_{k}}(k-1)+1$. In the same way, $M$ clustering and $M$ control rules can be reached, then the fuzzy system designed by this control rule is:

$$
f_{k}(x)=\frac{\sum_{l=1}^{M} A^{l}(k) \exp \left(-\frac{\left|x-x_{c}^{l}\right|^{2}}{\sigma}\right)}{\sum_{l=1}^{M} B^{l}(k) \exp \left(-\frac{\left|x-x_{c}^{l}\right|^{2}}{\sigma}\right)}
$$

In which, $A^{l}(k), B^{l}(k), \sigma$ are network parameters, $A^{l}(k)$ is the sum of the output value of input-output data in $l, B^{l}(k)$ is the amount of input-output data pair after $k$ data has been used, while $x_{c}^{l}$ is the clustering center of $l$.

Step 3: Return to step 2 on condition of $k=k+1$.

\subsection{Improving and Optimization}

Through the designed steps above, we conclude that:

(1) The number of rules in fuzzy systems depends on the input-output data complexity of the diameter of the fuzzy system. The smaller $r$ is the more control rules there are and the more complex the system; the bigger $r$ is the less control rules there are. Once the radius $r$ is set, there are no more changes, which inevitably affects the degree of shadow.

(2) In step 2, we only used the information of $x_{c}^{l}(l=1,2, \cdots)$ without considering the clustering of information contained in other samples.

(3) Besides, from formulae (4.1) and (4.2), we only consider the clustering number of $M$ and $M+1$, despite the number of samples in the clustering.

In view of these disadvantages, this paper proposes the following steps for improvement approaches.

Step 1: Calculate the distance among all the samples using $d_{i j}(i, j=1,2, \cdots, n)$, where $n$ is the sum of samples:

$$
d_{i j}=\sqrt{\left\|x_{0}^{i}-x_{0}^{j}\right\|^{2}+\left\|y_{0}^{i}-y_{0}^{j}\right\|^{2}}
$$

To calculate the average value $\bar{d}$ of the distance between the samples $d_{i j}$ :

$$
\bar{d}=\frac{2}{n(n-1)} \sum_{a=1}^{n-1} \sum_{b=a+1}^{n} d_{a b}
$$

To get the samples of $t$ close relating to sample $i$, we let the distance between $t$ and $i$ be $d_{i}^{1}, d_{i}^{2}, \cdots, d_{i}^{t}$ to calculate the average value of $\bar{d}_{i}$ :

$$
\bar{d}_{i}=\frac{1}{t} \sum_{p=1}^{t} d_{i}^{p},
$$

In which, $\bar{d}_{i}$ indirectly indicates the number of samples near the sample of $i$.

Step 2: Start from the first input-output data pair, supposing $x_{0}^{1}$ as the cluster center, and set $A^{1}(1)=y_{0}^{1}, B^{1}(1)=1$ and set $r$ as the radius of clustering.

Step 3: In consideration that the $k$ pair input-output data $\left(x_{0}^{k}, y_{0}^{k}\right), k=2,3, \cdots N$, there exists $M$ clustering that have centers as $x_{c}^{1}, x_{c}^{2}, \ldots \ldots x_{c}^{m}$ respectively. To calculate the distance from $x_{0}^{k}$ to $M$ cluster centers $\left|x_{0}^{k}-x_{c}^{m}\right|, m=1,2, \cdots M$, if the minimum distance among which is $\left|x_{0}^{k}-x_{c}^{p}\right|$, then $p \in(1,2, \cdots, M)$, that is, $x_{c}^{p}$ is the clustering of nearest neighbor principle of $x_{0}^{k}$. 
Step 4: Given that $r_{k}^{*}=\frac{r \bar{d}_{k}}{\bar{d}}$, if

(1) $\left|x_{0}^{k}-x_{c}^{p}\right|<r_{k}^{*}$, sample $\left(x_{0}^{k}, y_{0}^{k}\right)$ belongs to $p, A^{p}(k)=A^{p}(k)+y_{0}^{k}$, $B^{p}(k)=B^{p}(k)+1, \quad x^{p}(k)=x^{p}(k)+x_{0}^{k}, \quad$ in which, $x^{p}$ indicates $p$ and the sum of each input point of samples in it. Goto step 5.

(2) $\left|x_{0}^{k}-x_{c}^{p}\right|>r_{k}^{*}$, sample $\left(x_{0}^{k}, y_{0}^{k}\right)$ in a new cluster, $x_{0}^{k}$ is the new center, then $x_{c}^{M+1}(k)=x_{0}^{k}, A^{M+1}(k)=y_{0}^{k}, B^{M+1}(k)=1$ 。Return to step 2.

Step 5: Once the clusters are identified, the initial fuzzy rule base will be established. Entering the learning samples into the network, we will design a fuzzy logic system consisting of input, reasoning, and output. Through the adaptive law mentioned in this chapter to adjust the network weights, the corresponding parameters and control rules will be obtained.

Step 6: After the learning, the fuzzy system with the optimizing control rules is:

$$
f_{k}(x)=\frac{\sum_{l=1}^{M} A^{l}(k) \exp \left(-\frac{\left|x-x_{c}^{l_{c}}\right|^{2}}{\sigma}\right)}{\sum_{l=1}^{M} B^{l}(k) \exp \left(-\frac{\left|x-x_{c}^{l^{2}}\right|^{2}}{\sigma}\right)}
$$

In which, $A^{l}(k), B^{l}(k), \sigma$ are network parameters, $A^{l}(k)$ is the sum of the output value of input-output data in $l$.

\section{Simulation Example}

In view of the system:

$$
x(k+1)-x(k)=T_{s}\left\{x^{3}(k)+\exp [x(k)]+\cos x^{2}(k)+\left[1+\cos ^{2} x(k)\right] u(k)\right\}
$$

where $x(k)$ is the state variable, $u(k)$ is the control input, and sampling time is 0.01 second.

As for the system (23), it is estimated that the nonlinear dynamics is $\hat{f}=1$ and the control gain is $\hat{g}=1$. Apply the design method of the controller proposed in this chapter to select sliding mode $s=x(k)$, and to take the parameters $\alpha=-5, \beta=20, \omega_{1}=0.01, \omega_{2}=1$, and $\phi(0)=0.1$, in adaptive law (13). We get the fuzzy sets and rules corresponding to DFLS as in [18]. Choose one of the parameters of the fuzzy partition $\theta_{1}=2, \theta_{1}=50$. It is assumed that a non-tracking model, the tracking signal, is zero. Simulation results are shown in [18].

To validate the effect of taking the improved fuzzy rules optimization method, a DFLS fuzzy set partition and fuzzy rules are optimized. Figure 1. and Figure 2. show the optimized and non-optimized control signal and state response signal results. The curves show that the closed-loop system is stable, and the control effect is smooth enough.

Additionally, Figure 1. and Figure 2. indicate that rules optimization indeed improves the control performance. Optimal control effect in the system can respond more quickly. There is no chattering of $u_{v}$ and the robustness remains. The introduction of boundary layer guarantees that the sliding mode will reach quasi switching band. DFLS enables the control signal to have the filtering function, which eliminates the high frequency chattering. 


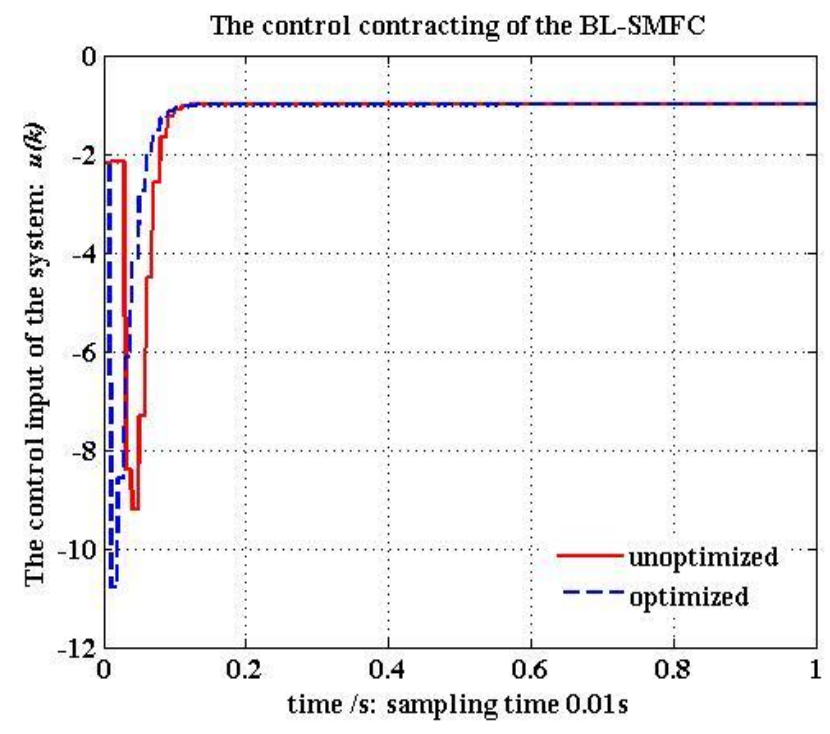

Figure 1. Simulation Curves of the Control Input of System (23)

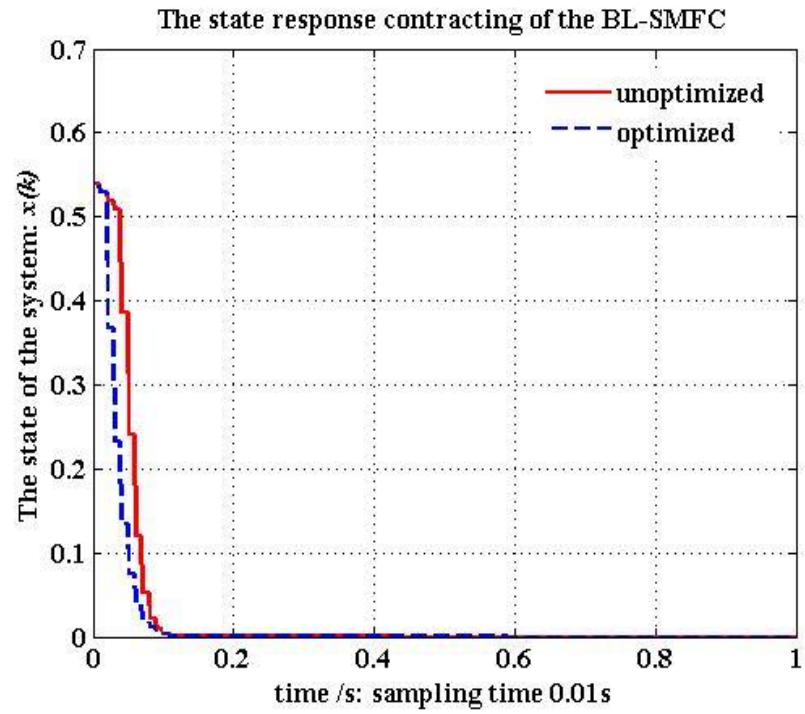

Figure 2. Simulation Curves of the State Responses of System (23)

\section{Conclusions}

This paper proposes an optimization AFSMC controller design with boundary layer self-tuning. The optimization method is applied to mend the rules and parameters of the dynamic fuzzy logic system (DFLS) which is equivalent to the pre-designed SMC control. Taking a kind of clusters meaning method, namely nearest neighbor algorithm, for the optimization of the FLS, the control performance is improved. Simulation contrast proved the correctness of the proposed design.

\section{Acknowledgments}

The authors gratefully acknowledge the helpful comments and suggestions of the Editors and the Reviewers, which have improved the presentation. 


\section{References}

[1] Gang Feng, "A Survey on Analysis and Design of Model-Based Fuzzy Control Systems", IEEE Transactions on Fuzzy System, vol. 14, no. 5, (2006), pp. 676-697.

[2] Hongyi Li, Jinyong Yu, Chris Hilton et al., "Adaptive Sliding-Mode Control for Nonlinear Active Suspension Vehicle Systems Using T-S Fuzzy Approach”, IEEE Transactions on Industrial Electronics, vol. 60, no. 8, (2013), pp. 3328-3338.

[3] Han Ho Choi, "Robust Stabilization of Uncertain Fuzzy-Time-Delay Systems Using Sliding-ModeControl Approach", IEEE Transactions on Fuzzy Systems, vol. 18, no. 5, (2010), pp. 979-984.

[4] Wallace M. Bessa, Max S. Dutra and Edwin Kreuzer, "Sliding Mode Control with Adaptive Fuzzy Dead-Zone Compensation of an Electro-hydraulic Servo-System", Journal of Intelligent \& Robotic Systems: Theory \& Application, vol. 58, no. 1, (2010), pp. 3-16.

[5] Ruey-Jing Lian and Jeen Lin, "Enhanced Adaptive Self-Organizing Fuzzy Sliding-Mode Controller For Robotic Motion Control", International Journal of Innovative Computing, Information and Control, vol. 7, no. 9, (2011), pp. 5459-5475.

[6] K. Saoudi and M. N. Harmas, "Enhanced Design of an Indirect Adaptive Fuzzy Sliding Mode Power System Stabilizer for Multi-Machine Power Systems", International Journal of Electrical Power and Energy Systems, vol. 54, no. 1, (2014), pp. 425-431.

[7] W. M. Bessa, A. S. De Paula and M. A. Savi, "Sliding Mode Control With Adaptive Fuzzy Dead-Zone Compensation For Uncertain Chaotic Systems", Nonlinear Dynamics, vol. 70, no. 3, (2012), pp. 19892001 .

[8] V. Nekoukar and A. Erfanian, "Adaptive Fuzzy Terminal Sliding Mode Control for a Class of MIMO Uncertain Nonlinear Systems", Fuzzy Sets and Systems, vol. 179, no. 1, (2011), pp. 34-49.

[9] T. Yoshimura, "Design of an Adaptive Fuzzy Sliding Mode Control for Uncertain Discrete-Time Nonlinear Systems Based on Noisy Measurements”, International Journal of Systems Science, vol. 47, no. 3, (2016), pp.617-630.

[10] P. C. Chen, C. W. Chen and W. L. Chiang, "Linear Matrix Inequality Conditions of Nonlinear Systems by Genetic Algorithm-based $H_{\infty}$ Adaptive Fuzzy Sliding Mode Controller", Journal of Vibration and Control, vol. 17, no. 2, (2011), pp. 163-173.

[11] Yesma Bendaha, Benyouness Mazari and Mustapha Benghanem, "Development of Fuzzy Sliding Mode Control Optimized by Genetic Algorithm for Induction Motor”, Przeglad Elektrotechniczny, vol. 86, no. 9, (2010), pp. 343-347.

[12] Mohammad Hassan Khooban and Mohammad Reza Soltanpour, "Swarm Optimization Tuned Fuzzy Sliding Mode Control Design for a Class of Nonlinear Systems in Presence of Uncertainties", Journal of Intelligent \& Fuzzy Systems: Applications in Engineering and Technology, vol. 24, no. 2, (2013), pp. 383-394.

[13] M. R. Soltanpour and M. H. Khooban, "A Particle Swarm Optimization Approach for Fuzzy Sliding Mode Control for Tracking the Robot Manipulator", Nonlinear Dynamics, vol. 74, no. 1, (2013), pp. 467-478.

[14] Taher Niknam, Mohammad Hassan Khooban, Abdollah Kavousifard and M. R. Soltanpour, "An Optimal Type II Fuzzy Sliding Mode Control Design for a Class of Nonlinear Systems", Nonlinear Dynamics, vol. 75, no. 1/2, (2014), pp. 73-83.

[15] C. G. Lhee, J. S. Park, H. S. Ahn and D. H. Kim, "Sliding-Like Fuzzy Logic Control with Self-Tuning the Dead Zone Parameters", Proceedings of IEEE International Fuzzy Systems Conference, Seoul, Korea, (1999), August 22-25, pp. 544-549.

[16] C. G. Lhee, J. S. Park, H. S. Ahn and D. H. Kim, "Sliding-Like Fuzzy Logic Control with Self-Tuning the Dead Zone Parameters", IEEE Transaction on Fuzzy Systems, vol. 9, (2001), pp. 343-348.

[17] Xiaoyu Zhang, "Adaptive sliding mode-like fuzzy logic control for nonlinear systems", Journal of Communication and Computer, vol.6, no.1, (2009), pp 53-60.

[18] Xiaoyu Zhang and Fang Guo, "Sliding Mode-Like Fuzzy Logic Control with Boundary Layer SelfTuning for Discrete Nonlinear Systems", Advances in Intelligent Systems and Computing, vol. 213, (2014), pp. 479-490. 


\section{Authors}

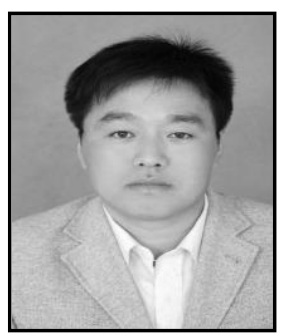

Jingxin Wei, was born in 1977. He received the B.S. degree from Liaoning Technical University in 2002, the M.S. degree from Communication University of China in 2008. Now he is a lecturer of North China Institute of Science and Technology. His research interests include network optimization, complex systems, and intelligent systems etc.

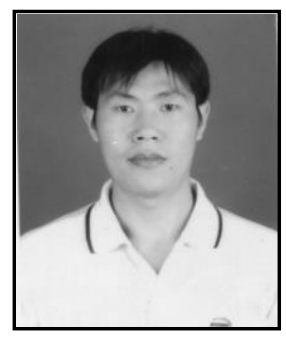

Xiaoyu Zhang, was born in 1978. He received the B.S. degree from Yanshan University in 2000, the M.S. and Ph.D. degree both from Zhejiang University in 2003 and 2006 respectively. He worked at Nanchang University as a lecturer from Jun. 2006 to Jun. 2007, worked at North China Institute of Science and Technology as a lecturer from Jul. 2007 to Dec. 2009, as an Associate Professor form Jan. 2010 to Dec. 2016. Now he is a Professor of North China Institute of Science and Technology. His research interests include nonlinear control, intelligent control, switched systems, adaptive control of nonlinear systems, analysis, synthesis and application of complex dynamic systems etc. 